\title{
Durability of pericardial versus porcine bioprosthetic heart valves
}

\author{
Gary L. Grunkemeier, PhD, ${ }^{\mathrm{a}}$ Anthony P. Furnary, MD, ${ }^{\mathrm{b}}$ YingXing Wu, MD, MS, ${ }^{\mathrm{a}}$ Lian Wang, MS, ${ }^{\mathrm{a}}$ and \\ Albert Starr, MD
}

Objectives: To compare the probability, and modes, of explantation for Carpentier-Edwards pericardial versus porcine valves.

\begin{abstract}
Methods: Our porcine series began in 1974 and our pericardial series in 1991, with annual prospective followup. We used the Kaplan-Meier method and Cox regression for estimation and analysis of patient mortality, and the cumulative incidence function and competing risks regression for estimation and analysis of valve durability.

Results: Through the end of 2010, we had implanted 506 porcine and 2449 pericardial aortic valves and 181 porcine and 163 pericardial mitral valves. The corresponding total and maximum follow-up years were 3471 and 24 , 11,517 and 18,864 and 22, and 645 and 9. The corresponding probabilities (cumulative incidence function) of any valve explant were $7 \%, 8 \%, 22 \%$, and $8 \%$, and of explant for structural valve deterioration were $4 \%, 5 \%$, $16 \%$, and $5 \%$ at 15 years for the first 3 series and at 8 years for the fourth (pericardial mitral valve) series. Using competing risks regression for structural valve deterioration explant, with age, gender, valve size, and concomitant coronary bypass surgery as covariates, a slight (subhazard ratio, 0.79 ), but nonsignificant, protective effect was found for the pericardial valve in the aortic position and a greater (subhazard ratio, 0.31) and almost significant $(P=.08)$ protective effect of the pericardial valve in the mitral position. Leaflet tear was responsible for $61 \%$ of the structural valve deterioration explants in the porcine series and $46 \%$ in the pericardial series.
\end{abstract}

Conclusions: Using competing risks regression, the pericardial valve had a subhazard ratio for structural valve deterioration explant of less than 1 in both positions, approaching statistical significance in the mitral position. The mode of structural valve deterioration was predominantly leaflet tear for porcine valves and fibrosis/ calcification for pericardial valves. (J Thorac Cardiovasc Surg 2012;144:1381-6)

We began using Carpentier-Edwards (CE) porcine valves in 1974 and then migrated to the CE pericardial valves when they became available (aortic in 1991 and mitral in 2000). Surgeon preference for the pericardial valves was determined from tests performed by Edwards Laboratories and Professor Carpentier, revealing greater in vitro durability of pericardium. In 2003, we compared our experience with CE aortic valve replacement (AVR) in 518 porcine valves implanted from 1974 to 1996 and 1021 pericardial valves implanted from 1991 to 2002, with a maximum follow-up of 18 and 10 years, respectively. ${ }^{1}$

We found that the 10-year Kaplan-Meier freedom from explantation was lower for porcine than for pericardial

\footnotetext{
From the Medical Data Research Center, ${ }^{\text {a }}$ Providence Health \& Services, Portland, Ore; Starr-Wood Cardiac Surgery Group, ${ }^{\mathrm{b}}$ St Vincent Hospital and Medical Center, Portland, Ore; and ${ }^{\mathrm{c}}$ Division of Cardiovascular Medicine, Oregon Health \& Science University, Portland, Ore.

This study was partially supported by a grant from Edwards Lifesciences.

Disclosures: Drs Starr and Furnary are consultants for Edwards Lifesciences. The other authors have nothing to disclose with regard to commercial support.

Read at the 38th Annual Meeting of The Western Thoracic Surgical Association, Maui, Hawaii, June 27-30, 2012.

Received for publication June 28, 2012; revisions received July 30, 2012; accepted for publication Aug 23, 2012; available ahead of print Sept 24, 2012.

Address for reprints: Lian Wang, MS, Medical Data Research Center, Providence Health \& Services, 9205 SW Barnes Rd, Suite 33, Portland, OR 97225 (E-mail:

Lian.Wang@providence.org).

$0022-5223 / \$ 36.00$

Copyright (c) 2012 by The American Association for Thoracic Surgery

http://dx.doi.org/10.1016/j.jtcvs.2012.08.060
}

valves $(90 \%$ vs $97 \%, P=.04)$, and concluded that, "The current CE pericardial valve offers better midterm durability than the traditional CE porcine valve. Its freedom from SVD [structural valve deterioration] and reoperation makes it our current bioprosthesis of choice for AVR in appropriately selected patients." 1

Dr Stuart Jamieson commented on our study, "Perhaps the most valuable conclusion from this study, and others similar to it, is that the durability of a tissue valve, in particular the durability of pericardial valves in the aortic position, is better than $90 \%$ at 10 years. However, the 10 -year test is relatively easy to pass. Relative freedom from structural deterioration at 20 years will be the important milestone." 2

We now have 8 years' additional follow-up. Although we have not yet achieved a 20-year estimate, we now have a fairly precise durability comparison for AVR at 15 years. In addition, we now also report on mitral valve replacement (MVR). Although we have far fewer MVRs, the mitral position is known to have a greater risk of structural valve deterioration (SVD) and, thus, similar to an accelerated fatigue tester, might provide a magnified comparison of any differences in durability.

\section{MATERIALS AND METHODS Clinical Material}

Through the end of 2010, we had performed 506 CE porcine and 2449 CE pericardial isolated AVRs and $181 \mathrm{CE}$ porcine and $163 \mathrm{CE}$ pericardial 

Abbreviations and Acronyms
AVR = aortic valve replacement
$\mathrm{CABG}=$ coronary artery bypass grafting
$\mathrm{CE}=$ Carpentier-Edwards
CIF = cumulative incidence function
$\mathrm{CRR}=$ competing risks regression
$\mathrm{KM}=$ Kaplan-Meier
MVR $=$ mitral valve replacement
SHR = subhazard ratio
SVD $=$ structural valve deterioration

isolated MVRs. By “isolated," we mean no concomitant or previous valve replacement or repair procedures performed in another position. Concomitant coronary artery bypass grafting (CABG) was not an exclusion criterion. The implant techniques were standardized for the entire series, with the same aortic incision, myocardial protection, and the same surgical group. There was an informative pattern to the distribution of patient age over time (Figure 1). A gradual increase occurred in the mean age with the porcine valve, as late SVD became known to be related to a younger age. Then, a gradual revisiting of younger patient ages occurred as additional experience with the pericardial valve was gained-and with it, the perception of improved durability.

Because our primary interest was in the long-term durability, we restricted the subsequent analysis to operative survivors only, with $469 \mathrm{CE}$ porcine and $2356 \mathrm{CE}$ pericardial isolated AVRs and $158 \mathrm{CE}$ porcine and 154 CE pericardial isolated MVRs (Table 1). Since 1960, we have prospectively interviewed all heart valve replacement patients at least annually for their entire life. At the end of 2010, the maximum follow-up for these 4 patient series ranged from 9 to 24 years (Table 1). We considered valve patients lost to follow-up if they were not known to be dead or to have had the valve explanted and had not responded to interview attempts for 2 years.

Because the present project involved only the study of existing records and used only de-identified data, it qualified for exemption from institutional review board approval, according to Exemption 45 CFR 46.101(b)(4).

\section{Statistical Analysis}

For the analysis of mortality, we use the Kaplan-Meier (KM) method ${ }^{3}$ and Cox regression analysis. ${ }^{4}$ For the analysis of valve explantation, we used the cumulative incidence function (CIF), the appropriate method to describe events with competing risks (death was considered a competing risk, because it precludes the possibility of a future explant). The CIF estimates the probability of a valve actually requiring explantation — before the patient dies. Analogous to the use of Cox regression for multivariate analysis of risk factors for death, competing risks regression (CRR) was used for the analysis of explantation. Instead of computing hazard ratios, such as would occur with Cox regression analysis, CRR computes subhazard ratios (SHRs), because the CIF is a subdistribution and not a complete distribution. This is because the probability of valve explant will not achieve $100 \%$, as will the probability of patient death. The statistical packages used in this analysis included R (R Foundation for Statistical Computing, Vienna, Austria) and Stata (StataCorp, College Station, Tex): all figures were produced using $\mathrm{R}$, including the local regression (LOESS) nonparametric regression curves. See the Appendix for a discussion of the competing risks and software options for computing CIF and CRR.

\section{RESULTS}

\section{Patient Mortality}

Unadjusted late mortality was lower for pericardial than for porcine valves, in both AVR and MVR, although the

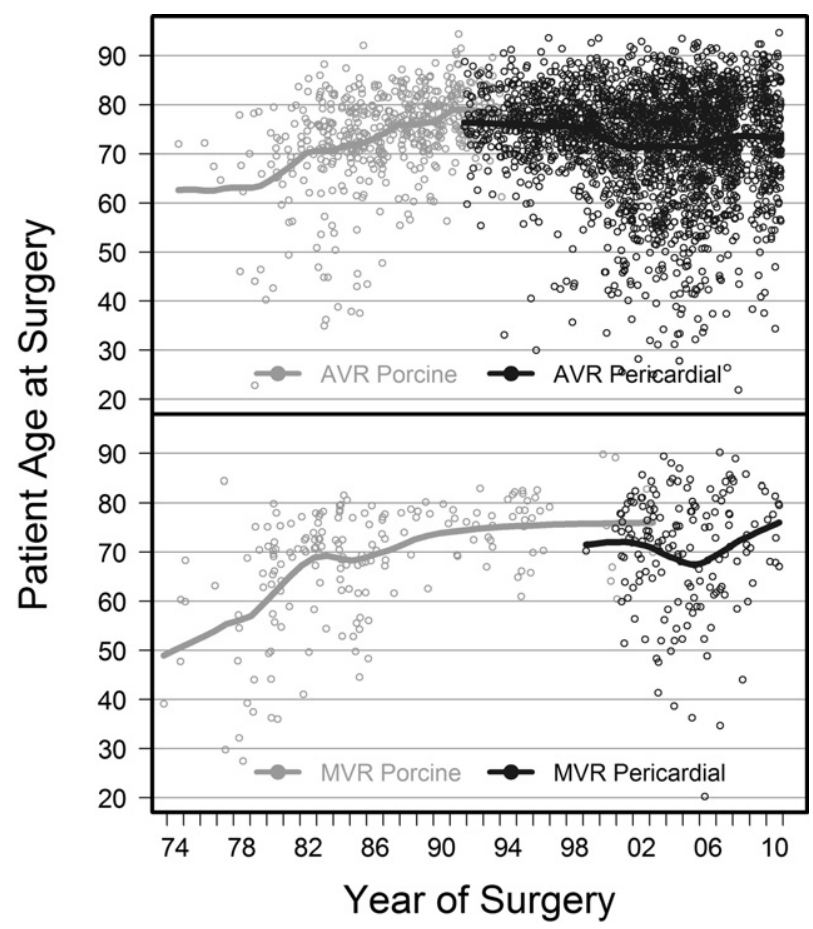

FIGURE 1. Scatter plot of patient age during implant year, with local regression (LOESS; locally weighted scatterplot smoothing) nonparametric regression curves fit to the individual points. $A V R$, Aortic valve replacement; $M V R$, mitral valve replacement.

numeric differences were not great: $81 \%$ versus $88 \%$ at 15 years for $\operatorname{AVR}(P=.02)$ and $50 \%$ versus $57 \%$ at 8 years for MVR $(P=.04)$. Separate Cox regression analyses of late survival for AVR and MVR - using valve type, patient age at implantation, gender, CABG, and valve size (Table 2)showed the pericardial valve to be protective (hazard ratio $<1)$ for mortality, significantly for $\operatorname{MVR}(P=.003)$ but not quite for $\operatorname{AVR}(P=.054)$.

\section{Valve Explantation}

Our main interests in the present study were the endpoints of overall explantation and explantation for SVD. All explants. For each position, the porcine valve had a greater probability (CIF) of overall explantation than the pericardial valve at 10 years (Figure 2). However, the probabilities of valve explantation by 15 years were similar in the aortic position. The CIF probability of explantation by 15 years after porcine and pericardial AVR was $7 \% \pm$ $1.3 \%$ (standard error [SE]) and $8 \% \pm 1.2 \%$ (Figure 2 ), respectively $(P=.12$, using univariate $\mathrm{CRR})$. The probabilities of explantation were $22 \% \pm 3.7 \%$ by 15 years for porcine MVR and $8 \% \pm 3.8 \%$ by 8 years for pericardial $\operatorname{MVR}(P=.03$, using univariate CRR).

Explants for SVD. The CIF probability of explantation for SVD by 15 years after porcine and pericardial AVR was $4 \%$ $\pm 1.0 \%$ (SE) and $5 \% \pm 0.9 \%$ (Figure 3 ), respectively 
TABLE 1. Clinical material for operative survivors

\begin{tabular}{|c|c|c|c|c|c|c|}
\hline & \multicolumn{3}{|c|}{ Aortic replacement } & \multicolumn{3}{|c|}{ Mitral replacement } \\
\hline & Pericardial & Porcine & $P$ value & Pericardial & Porcine & $P$ value \\
\hline Implant years & $1991-2010$ & 1976-1994 & & $2000-2010$ & $1974-2003$ & \\
\hline Valves (n) & 2356 & 469 & & 154 & 158 & \\
\hline Mean age & 72.9 & 73.8 & .08 & 70.4 & 68.0 & .09 \\
\hline Male $(\%)$ & 62.5 & 61.6 & .73 & 57.1 & 39.2 & $<.01$ \\
\hline Mean valve size $(\mathrm{mm})$ & 24.1 & 23.4 & $<.01$ & 29.2 & 30.4 & $<.01$ \\
\hline CABG $(\%)$ & 48.2 & 38.8 & $<.01$ & 37.7 & 31.0 & .22 \\
\hline \multicolumn{7}{|l|}{ Follow-up } \\
\hline Patient-years & 11,514 & 3470 & & 645 & 863 & \\
\hline Mean (y) & 4.9 & 7.4 & & 4.2 & 5.5 & \\
\hline Maximum (y) & 18 & 24 & & 9 & 22 & \\
\hline Completeness (\%) & 94 & 87 & & 93 & 87 & \\
\hline
\end{tabular}

$C A B G$, Coronary artery bypass grafting.

$(P=.35$, using univariate $\mathrm{CRR})$. The probabilities of explant for SVD were $16 \% \pm 3.3 \%$ (SE) by 15 years for porcine MVR and $5 \% \pm 3.2 \%$ by 8 years for pericardial MVR $(P=.13$, using univariate $\mathrm{CRR})$. Using CRR for risk adjustment, with age, gender, valve size, and CABG as covariates, the pericardial valve was protective $(\mathrm{SHR}<1)$ for SVD explant in both positions (Table 3). The difference was almost statistically significant $(P=.08)$ in the mitral position $(\mathrm{SHR}=0.31)$ but was not in the aortic position $(\mathrm{SHR}=$ 0.79). Age was a highly significant protective factor for SVD explant in both positions $(P<.001)$; for AVR, CABG was almost significantly protective $(P=.07)$. The mode of failure was different between the 2 valve types: the numbers of leaflet tears and fibrosis/calcification were 23 and $15(61 \%$ were leaflet tears) for the porcine series (AVR and MVR combined) and 11 and 13 (46\% were leaflet tears) for the combined pericardial series.

\section{DISCUSSION}

In our previous report, ${ }^{1}$ only 6 aortic pericardial valves had been in place longer than 10 years. We now have 213 such patients, and the AVR durability advantage we previously observed at 10 years seemed to be attenuated at 15 years (Figure 2). Dr Jamieson's comment ${ }^{2}$ was that $90 \%$ durability at 10 years is fairly easy to pass, but the challenge

TABLE 2. Multivariate Cox regression of late mortality with $\mathrm{CE}$ tissue valves

\begin{tabular}{llrlllr}
\hline & \multicolumn{2}{c}{ AVR } & & \multicolumn{2}{c}{ MVR } \\
\cline { 2 - 3 } \cline { 5 - 6 } \multicolumn{1}{c}{ Risk factor } & HR $(\mathbf{9 5} \% \mathbf{C I})$ & $\boldsymbol{P}$ value & & HR $(\mathbf{9 5} \% \mathbf{C I})$ & $\boldsymbol{P}$ value \\
\hline Pericardial valve & $0.88(0.774-1.00)$ & .054 & & $0.53(0.34-0.81)$ & .003 \\
Patient age & $1.05(1.04-1.06)$ & $<.001$ & & $1.05(1.03-1.08)$ & $<.001$ \\
Male gender & $1.22(1.05-1.42)$ & .011 & & $1.14(0.79-1.64)$ & .486 \\
CABG & $1.13(1.00-1.27)$ & .045 & & $1.42(0.99-2.01)$ & .052 \\
Valve size & $0.96(0.92-1.00)$ & .028 & & $0.97(0.88-1.06)$ & .468 \\
\hline
\end{tabular}

$A V R$, Aortic valve replacement; $C A B G$, coronary artery bypass grafting; $C E$, Carpentier-Edwards; $C I$, confidence interval; $H R$, hazard ratio; $M V R$, mitral valve replacement. would be for 20 years. The CIF for explantation was still lower than $10 \%$ at 15 years in the aortic position, for both valve types (Figure 2). Thus, we continue to monitor these patients, and await our 20-year results.

A recent innovative study, fueled by a meta-analysis ( 8 reports, 2685 patients, 12,250 patient-years) of AVR with CE pericardial valves, ${ }^{5}$ used computer simulations to calculate "...the 'actual' or lifetime risks of valve-related events and reoperation after valve implantation." This method simulates the various states of mortality and morbidity that a valve might be in at any time after implantation and conforms exactly to the concept of CIF, rather than the KM method, to estimate SVD. The investigators found that "the lifetime risk of a reoperation due to SVD reduced with advancing age of implantation and was $17 \%$ and $5 \%$, respectively, for 65 - and 75-year-old males." This agrees well with our finding of $5 \%$ SVD explantation at 15 years for our aortic CE pericardial patients, who had an average age of 73 years.

Other aortic porcine valves have been found to have excellent long-term durability, including the third-generation

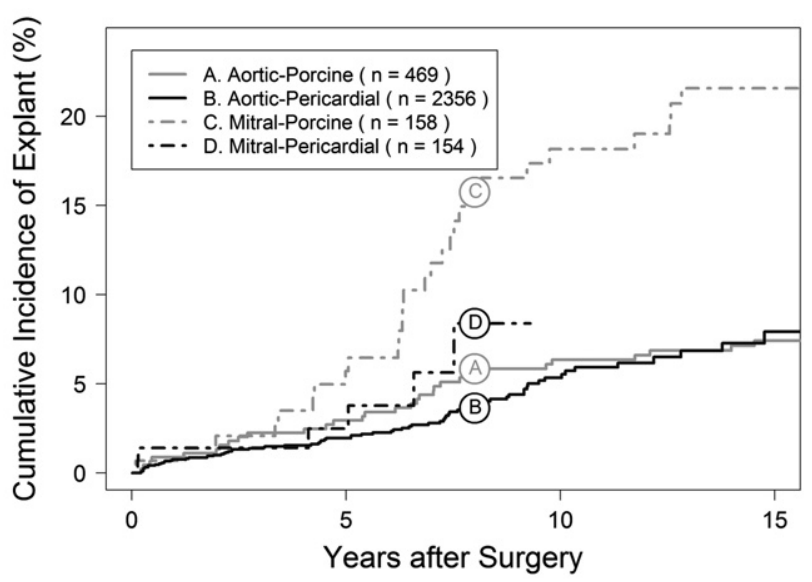

FIGURE 2. Cumulative incidence function estimates of explant for any reason in operative survivors. 


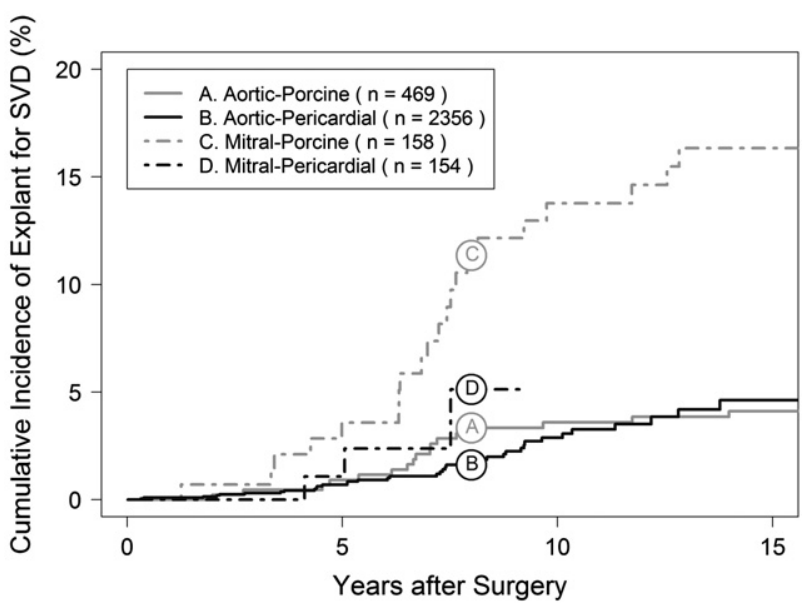

FIGURE 3. Cumulative incidence function estimates of explant for structural valve deterioration $(S V D)$ in operative survivors.

porcine Hancock II (Medtronic Inc, Minneapolis, Minn). ${ }^{6}$ The KM percentage of reoperation for SVD by 15 years was $12 \% \pm 1.8 \%$ (SE). This was somewhat greater than our values of $4 \%$ and $5 \%$; however, these patients were younger (67 vs 73 years), and the KM method produces greater failure rates than the CIF method (see the Appendix).

A very recent observational study of pericardial versus porcine AVR that concentrated on survival concluded that "Despite the better hemodynamic performance documented in prior investigations, pericardial valves do not confer any survival advantage over porcine valves in patients aged 65 years or older undergoing aortic valve replacement." ${ }^{, 7}$ These investigators observed a slight survival advantage for porcine compared with pericardial valves. We found a slight risk-adjusted advantage, although not quite significant $(P=.054)$, in the survival of pericardial versus porcine valves in the aortic position (Table 2). However, the numeric difference was relatively small $(7 \%$ at 15 years). One reason for the discrepancy could have been because our AVR porcine cases were performed in an earlier, almost nonoverlapping, implant era, compared with theirs (1974-1994 vs 1993-2007).

\section{Study Limitations}

It is always challenging to draw data-supported conclusions from observational series. The major issue with nonrandomized comparisons is the possibility of selection bias. Although the valve types we studied were not randomized, they were also not concurrent. Rather, the 2 valve types were used consecutively (Figure 1); thus, the selection bias should have been minimal, because each valve type was used in virtually all tissue valve recipients during its era of use. However, this does implicate as possible confounders other variables that changed systematically between the
TABLE 3. Multivariate competing risks regression of explantation for SVD with CE tissue valves

\begin{tabular}{lcrlll}
\hline & \multicolumn{2}{c}{ AVR } & & \multicolumn{2}{c}{ MVR } \\
\cline { 2 - 3 } \cline { 6 - 6 } \multicolumn{1}{c}{ Risk factor } & SHR $(\mathbf{9 5} \%$ CI $)$ & $\boldsymbol{P}$ value & & SHR $(\mathbf{9 5} \%$ CI $)$ & $\boldsymbol{P}$ value \\
\hline Pericardial valve & $0.79(0.43-1.45)$ & .448 & & $0.31(0.08-1.13)$ & .075 \\
Patient age & $0.94(0.92-0.95)$ & $<.001$ & & $0.93(0.90-0.95)$ & $<.001$ \\
Male gender & $1.39(0.62-3.14)$ & .422 & & $0.40(0.14-1.16)$ & .092 \\
CABG & $0.50(0.24-1.05)$ & .069 & & $1.29(0.50-3.34)$ & .656 \\
Valve size & $0.96(0.82-1.12)$ & .608 & & $1.04(0.84-1.29)$ & .762 \\
\hline
\end{tabular}

$A V R$, Aortic valve replacement; $C A B G$, coronary artery bypass grafting; $C E$, Carpentier-Edwards; $C I$, confidence interval; $M V R$, mitral valve replacement; $S H R$, subhazard ratio; $S V D$, structural valve deterioration.

2 implant eras. We used only 4 factors for risk adjustment (Tables 2 and 3), and many other patient risk factors could be considered in the risk adjustment of a comparison of observational data such as ours. However, age is the most significant factor known to affect tissue valve durability and was accounted for in the analysis.

Although the CIF method is appropriate for estimating the probability of nonfatal events, a comparison between the CIFs of different valves can be problematic if the death rates are dissimilar, because a greater death rate will tend to reduce the CIF of valve explantation. However, in these series, the mortality was greater with the porcine than with the pericardial valves (Table 2). Thus, the lower mortality for the pericardial valve tended to increase the CIF of SVD explants, relative to the porcine valve. If this were not the case, it would only have increased the observed SVD explant advantage of the pericardial valve.

A criticism of the present study could be that the CE porcine valves we studied were first generation, and the pericardial valves have been evolving recently. However, most of the pericardial valves in the present study were also first generation, with the newer Magna version in predominant use only in the last 2 years of our study. Specifically, in the present study, $91 \%$ of the pericardial aortic valves were the original model $2700,95 \%$ of the pericardial mitral valves were model $6900,99.8 \%$ of the porcine aortic valves were the original model 2625 , and $93 \%$ of the porcine mitral valves were the original model 6625 . It will take some time to determine the relative performance of the latest generations of porcine and pericardial valves.

\section{CONCLUSIONS}

Using CRR, the CE pericardial valve had a SHR for SVD explant of less than 1 in both positions compared with the CE porcine valve. The difference was nearly statistically significant for MVR. The mode of SVD was predominantly leaflet tear for the porcine valve and fibrosis/calcification for the pericardial valve.

\section{References}

1. Gao G, Wu Y, Grunkemeier GL, Furnary AP, Starr A. Durability of pericardial versus porcine aortic valves. J Am Coll Cardiol. 2004;44:384-8. 
2. Jamieson SW, Madani MM. The choice of valve protheses. J Am Coll Cardiol. 2004;44:389-90.

3. Kaplan EL, Meier P. Nonparametric estimation from incomplete observations. J Am Stat Assoc. 1958;53:457-81.

4. Cox DR. Regression models and life tables. J Royal Stat Society Series B. 1972; 34:187-220.

5. Puvimanasinghe JP, Takkenberg JJ, Eijkemans MJ, Steyerberg EW, van Herwerden LA, Grunkemeier GL, et al. Prognosis after aortic valve replacement with the Carpentier-Edwards pericardial valve: use of microsimulation. Ann Thorac Surg. 2005;80:825-31.

6. David TE, Armstrong S, Maganti M. Hancock II bioprosthesis for aortic valve replacement: the gold standard of bioprosthetic valves durability? Ann Thorac Surg. 2010;90:775-81.

7. Said SM, Ashikhmina E, Greason KL, Suri RM, Park SJ, Daly RC, et al. Do pericardial bioprostheses improve outcome of elderly patients undergoing aortic valve replacement? Ann Thorac Surg. 2012;93:1868-75.

8. Andersen PK, Geskus RB, de Witte T, Putter H. Competing risks in epidemiology: possibilities and pitfalls. Int J Epidemiol. 2012;41:861-70.

9. Koller MT, Raatz H, Steyerberg EW, Wolbers M. Competing risks and the clinical community: irrelevance or ignorance? Stat Med. 2012;31:1089-97.

10. Putter H. Special issue about competing risks and multi-state models. J Stat Software. 2011;38:1-4.

11. Southern DA, Faris PD, Brant R, Galbraith PD, Norris CM, Knudtson ML, et al. Kaplan-Meier methods yielded misleading results in competing risk scenarios. J Clin Epidemiol. 2006;59:1110-4.

12. Scheike TH, Zhang MJ. Flexible competing risks regression modeling and goodness-of-fit. Lifetime Data Anal. 2008;14:464-83.

13. Putter H, Fiocco M, Geskus RB. Tutorial in biostatistics: competing risks and multi-state models. Stat Med. 2007;26:2389-430.

14. Fine JP, Gray RJ. A proportional hazards model for the subdistribution of a competing risk. J Am Stat Assoc. 1999;94:496-509.

\section{APPENDIX. ESTIMATORS OF BIOPROSTHESIS DURABILITY (CIF) VERSUS PATIENT SURVIVAL (KM)}

In this Appendix, we provide the justification for the use of the cumulative incidence function (CIF) as an estimator of the probability of valve explantation. This method is less well known than the widely used Kaplan-Meier (KM) estimate, ${ }^{3}$ which produces a survival (or mortality) curve in the presence of censoring (ie, before all the individuals in a series have died). The true mortality curve-after all patients have died-would be the cumulative deaths plotted versus time. This curve would increase at each death, by an amount equal to the percentage of the whole population that had died at that time, increasing from $0 \%$ to $100 \%$ at the last death (ie, the curve that the KM attempts to approximate for an ongoing series, before all patients have died).

For a nonfatal event, such as valve explantation, the corresponding estimate is the CIF, which estimates the percentage explanted over time in the presence of censoring (ie, before all the valves have ceased functioning). The true explant curve-after all the valves have ceased functioning (because of valve explantation or patient death)—would be the cumulative explants plotted versus time. This curve would increase with each explant by an amount equal to the percentage of the whole population explanted at that point, increasing from $0 \%$ to a percentage less than $100 \%$, at the point of the last explant (ie, the curve that the CIF attempts to approximate for an ongoing series).
The KM method is often used to estimate the incidence of explantation; however, this overestimates the true explant incidence. $^{8-12}$ To illustrate this, we used both AVR series combined (Figure 4). Each valve can be in 1 of 3 states at any point after surgery. From bottom to top in Figure 4, these states are (1) valve function terminated by explantation; (2) valve function terminated by patient death; and (3) valve still functioning. The lowest curve in Figure 4 is the CIF estimate of explantation. For comparison, the KM estimate of explantation is superimposed as a dashed line, and can be seen to overestimate the percentage of explants that will actually occur.

What does the KM curve estimate? First, it only works if death and explantation are independent, ${ }^{13}$ which in these situations is a false assumption, because younger patients will have lower death rates and greater structural valve deterioration rates than older patients. If explantation and death were independent, the KM method would estimate the theoretical durability of the valve in the event that no patient died. ${ }^{13} \mathrm{~A}$ thought experiment might clarify this idea. Imagine we used a biologic valve in 2 series of identical patients. The first group is given a magic elixir that prevents death - until after the tissue valve has failed and been explanted. The KM curve would provide the estimate of the explant curve in that series. The elixir is withheld from the second group, and death takes its usual course. The CIF curve would provide the estimate of the resulting failure/ explant curve, which would be lower than the previous KM curve. Thus, the KM curve does not provide a true probability of explantation, because most patients will die before their valve fails. The CIF takes into account the

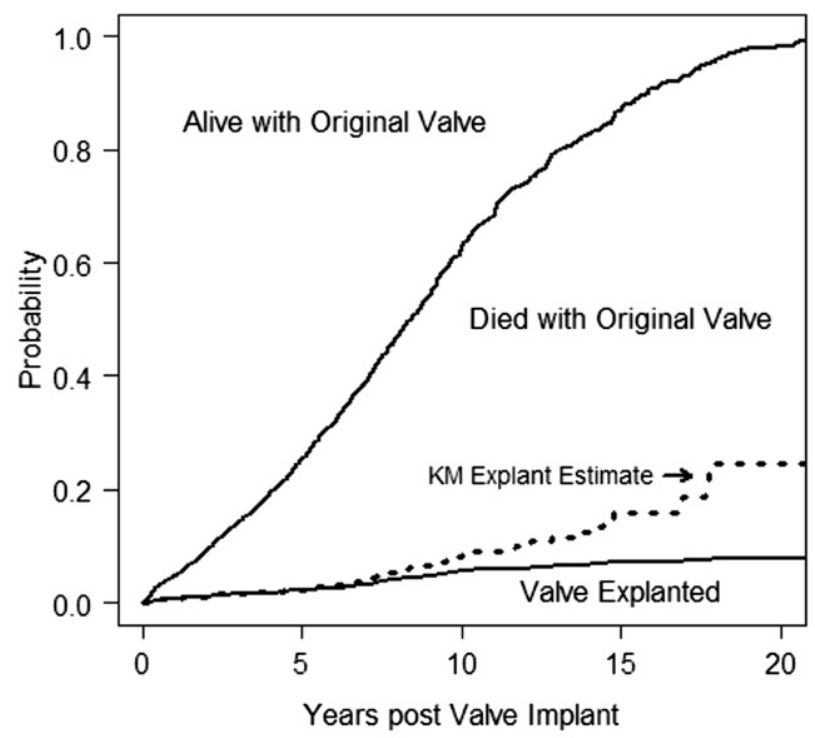

FIGURE 4. Kaplan-Meier ( $K M)$ estimate of explantation, superimposed on the distribution of multiple valve states over time for the porcine and pericardial aortic valve replacement series combined. 
mortality of the AVR recipients and, thus, provides a true estimate of the probability of the occurrence of explantation.

A patient cares less about the theoretical durability of the valve than about the "practical" durability of the valve (ie, will it last long enough to keep me alive until I die of some other cause?). However, we must focus not only on such overall values, but also on estimates that are individualized to a patient, taking into consideration other variables, especially age. Thus, from a patient standpoint, it is clear that the risk of structural valve deterioration should be balanced against the risk of survival. Similar to Cox regression analysis for mortality, the method of competing risks regression
(CRR) provides the appropriate patient-specific estimates for explantation and structural valve deterioration.

Software has been developed for the implementation of the CIF and CRR. For R (R Foundation for Statistical Computing, Vienna, Austria), from the cmprsk package, the function cuminc implements CIF and the function crr implements the CRR model of Fine and Gray. ${ }^{14}$ In the timereg package, the function comp.risk for CRR is based on Scheike and Zhang, ${ }^{12}$ and it contains the Fine and Gray model as a special case. For Stata (StataCorp, College Station, Tex), the command stcompet produces the CIF; for CRR, stcrreg implements the Fine and Gray method, ${ }^{14}$ and stcompadj is based on the Cox model. ${ }^{4}$ 